\title{
Effects of pre-consultation lists on the communicative behavior of children with cancer and their caregivers
}

\author{
Efeitos de listas de pré-consulta sobre \\ comportamento comunicativo de crianças \\ com câncer e seus cuidadores
}

\author{
Marina KOHLSDORF ${ }^{1}$ \\ Áderson Luiz COSTA JUNIOR' \\ Felipe Diniz MARQUES'
}

\begin{abstract}
This cross-sectional study present effects of pre-consultation lists on caregivers' and children's communicative behavior. Thirty-two dyads caregiver-child took part in this research, divided in three groups concerning the study design: Baseline 1, Intervention phase, and Baseline 2. Children and caregivers were individually interviewed before consultations on pediatric cancer treatment, in order to list doubts, expectations, and estimated issues to the medical visit. These themes were written down on a sheet of paper, attached to the child's medical record cover, and would then be used as a memo in the following consultation. Results show that pre-consultation lists produced no effects on children's behavior, however the procedure was associated to specific doubts discussed by caregivers regarding dietary recommendations, coping with side effects, child development, biological aspects of cancer, and school activities. This study presents a low cost procedure that may contribute to tailor communication in pediatric settings.
\end{abstract}

Keywords: Child; Child health; Neoplasm; Pediatrics.

\section{Resumo}

Este estudo transversal apresenta efeitos de listas de pré-consulta sobre comportamento comunicativo de cuidadores e crianças. Participaram 32 díades cuidador-criança, as quais foram divididas em três grupos de acordo com o delineamento do estudo: Linha de Base 1, Intervenção e Linha de Base 2. Crianças e cuidadores foram entrevistados individualmente antes das consultas em tratamento de câncer infantil para listar dúvidas, expectativas e assuntos estimados à consulta médica. Esses temas foram escritos em uma folha de papel anexada à capa do prontuário da criança, utilizada como lembrete para a consulta que se seguiria. Resultados mostram que as listas de pré-consulta não produziram efeitos no comportamento infantil, porém o procedimento foi associado a dúvidas específicas discutidas pelos cuidadores, relativas às recomendações alimentares, a lidar com efeitos colaterais, ao desenvolvimento da criança, aos aspectos biológicos do câncer e às atividades escolares. Este trabalho apresenta procedimento de baixo custo que pode contribuir para a comunicação sob medida em contextos pediátricos.

Palavras-chave: Criança; Saúde da criança; Neoplasia; Pediatria.

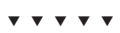

1 Universidade de Brasília, Instituto de Psicologia, Laboratório de Desenvolvimento em Condições Adversas. Campus Universitário Darcy Ribeiro, Ala Sul, Sala 025, SEQN 608 lote único, 70910-900, Brasília, DF, Brasil. Correspondência para/Correspondence to: M. KOHLSDORF. E-mail: <marinak@unb.br>. 
The study of communication in pediatric assistance has become more emphasized over the last two decades, considering the influence of interaction during consultations regarding self-care behaviors, quality of life, adaptation to treatment, satisfaction with health services, recalling of guidelines, better symptoms and physiological responses, management of stressors concerning health processes, better social support, adherence to treatment and fewer ambulatory returns (Ammentorp, Kofoed, \& Laulund, 2011; Clark et al., 2000; Cohen \& Wambolt, 2000; Coyne \& Gallagher, 2011; Croom et al., 2011; Crossley \& Davies, 2005; DiMatteo, 2004; Drotar, 2009; Howells \& Lopez, 2008; Nobile \& Drotar, 2003; Sleath et al., 2012).

Health communication may be perceived as a relational process of exchanging information and its understanding between at least two people, including contextual variables and individual characteristics (Araújo \& Cardoso, 2007; Arora, 2003; Fisher \& Broome, 2011; Wassmer et al., 2004). In some cases of health services, such as pediatric assistance, communication occurs necessarily between the health professional, patient and caregiver, a triadic interaction that requires specific abilities from pediatricians (Gabe, Olumide, \& Bury, 2004; Howells, Davies, \& Silverman, 2006; Howells \& Lopez, 2008; McGraw et al., 2012; Nobile \& Drotar, 2003; Tates \& Meeuwesen, 2001).

Although children's participation has been increasing, these patients remain excluded from interaction and their participation is restricted to 2 to $14 \%$ of the communicative process (Tates \& Meeuwesen, 2001) during small talk or when providing basic information (Coyne, 2008; Hallström, 2004; Nobile \& Drotar, 2003; Nova, Vegni, \& Moja, 2005; Rotenberg et al., 2008; Tates, Elbers, Meeuwesen, \& Bensing, 2002a; Tates, Meeuwesen, Elbers, \& Bensing, 2002b; Van Dulmen, 2004; Van Dulmen \& Holl, 2000; Vaknin \& Zisk-Rony, 2010; Wissow \& Kimel, 2002). However, four-year-old children already understand basic information related to self care, identify symptoms, have doubts, experience emotional issues related to the treatment and may feel guilty about the disease (Buckley \& Savage, 2010; Gordon et al., 2010; Knighting, Rowa-Dewar, Malcolm, Kearney, \& Gibson, 2010; Märtenson \& Fägerskiöld, 2007; Märtenson, Fägerskiöld, \& Berteró, 2007; Nova et al., 2005; Vatne, Slaughter, \& Ruland, 2010).

Another relevant aspect highlighted by the literature are the individual preferences for the kind and amount of information, which is related to the adaptation to treatment and satisfaction with health services, endorsing the need for tailored interventions that may contribute to the development of better interactions between pediatricians, caregivers, and children (Aburn \& Gott, 2011; Coyne \& Gallagher, 2011; Coyne \& Harder, 2011; Howells \& Lopez, 2008; Lambert, Glacken, \& McCarron, 2011; Ringnér, Jansson, \& Graneheim, 2010; Zwaanswijk et al., 2011). Therefore, psychosocial interventions are important in order to understand the triadic interaction and provide better communication in pediatric settings. Some studies have investigated the effects of surveys and questionnaires, which filled out by caregivers and/or pediatricians before consultations, on subsequent triadic communication. In studies conducted by Felt and O'Connor (2003) and Hayutin, Reed-Knight, Blount, Lewis, and McCormick (2009), this procedure promoted the discussion of psychosocial issues during medical visits and better satisfaction related to the health service. These authors emphasize that pre-consultation lists and questionnaires, filled out by caregivers before medical visits, may contribute to better communication with pediatricians, although children did not participate directly in these surveys.

Therefore, the main objective of this study was to analyze the functional effects of preconsultation surveys, filled out before medical visits and available during consultations, on the communicative behaviors of caregivers and children. This study is part of a larger research that investigated the effects of pre-consultation lists on several behavioral classes; in this paper, we focus on the communicative behaviors of caregivers and children. 
Method

\section{Participants}

This study was divided into three phases: Baseline 1, Intervention and Baseline 2. A total of 50 child-caregiver dyads in the first semester of cancer treatment were asked to participate in the research, but three refused and 15 were excluded as they could not be contacted after the first meeting. Therefore, a total of 30 mothers and two grandmothers participated in these three phases. Inclusion criteria were children's age (between the age of four and twelve years), caregiver's age (above the age of 18 years), and child-caregiver dyads in the first semester of pediatric cancer treatment. Exclusion criteria were children with other chronic health conditions, cancer relapse or sequelae, speaking difficulties, or those not responding to treatment. These 32 child-caregiver dyads were accompanied by and intermittently consulted with two pediatricians throughout the study, who had been working as regular staff in the pediatric cancer hospital for at least five years, aged 49 and 32 years with 26 and seven years of medical practice, respectively. Table 1 shows the characteristics of the 32 child-caregiver dyads that took part in this study.

\section{Instruments}

During all three phases, the consultations were recorded using two digital recorders and were also closely observed by the researcher who used an observational cursive protocol, developed exclusively for this study, on which contextual aspects of communication would be noted (child's voice intonation when crying or protesting, child's or caregiver's facial expressions of worry, sadness and tension, or when a participant left the room).

A pre-consultation survey, used only during the Intervention phase, was developed for this research based on the study of Zannon, Pereira, Arruda, Kohlsdorf, and Rocha (2002). The protocol had specific spaces for recording the following: (a) diagnosis and expectancies related to the treatment; (b) adherence to medication, self care and recommendations; (c) doubts and difficulties related to treatment; (d) estimated issues that caregiver and/or child would like to discuss with the physician.

Table 1

Participants of the study

\begin{tabular}{|c|c|c|c|c|}
\hline \multirow{2}{*}{ Characteristics } & \multicolumn{3}{|c|}{ Frequency } & \multirow{2}{*}{ Total } \\
\hline & Baseline 1 & Intervention & Baseline 2 & \\
\hline Children's ages* & 8 Dyads & 20 Dyads & 4 Dyads & \\
\hline 4 to 8 years & 7 & 11 & 3 & 21 \\
\hline 8 to 12 years & 1 & 9 & 1 & 11 \\
\hline Average $(S D)$ & $5.5(0.92)$ & $8.5(2.87)$ & $6.94(1.67)$ & $7.5(2.7)$ \\
\hline \multicolumn{5}{|l|}{ Diagnosis } \\
\hline Leukemia or lymphoma & 5 & 14 & 3 & 22 \\
\hline Solid tumors & 3 & 6 & 1 & 10 \\
\hline \multicolumn{5}{|l|}{ Caregivers' ages } \\
\hline 20 to 35 years & 5 & 10 & 1 & 16 \\
\hline Above 35 years & 3 & 10 & 3 & 16 \\
\hline Average $(S D)$ & $32(7.4)$ & $35(7.3)$ & $37(8.4)$ & $35(7.4)$ \\
\hline \multicolumn{5}{|l|}{ Time since diagnosis* } \\
\hline 1 month & - & 7 & - & 7 \\
\hline 2 to 4 months & 6 & 11 & 3 & 20 \\
\hline 4 to 6 months & 2 & 2 & 1 & 5 \\
\hline Average $(S D)$ & $2.5(1.01)$ & $1.68(1.37)$ & $2.34(1.08)$ & $2.03(1.36)$ \\
\hline
\end{tabular}

Note: ${ }^{*} p \leq 0.05$.

SD: Standard Deviation. 


\section{Procedures}

This study was first approved by Ethics Committee in Health, Education and Science Foundation in the Federal District (Fundação de Ensino e Pesquisa Ciências da Saúde/Distrito Federal), under register 301/09, number 289/2009. All audio recordings are stored in a passwordprotected confidential file. The research was conducted in a hospital that receives children and adolescents for cancer treatment. All participants physicians, caregivers and children, after their parents' consent, who had their consultations recorded -, were first approached and asked to participate and signed a form of Informed Consent. On the first meeting, the characteristics of the childcaregiver dyads were obtained verbally.

This cross-sectional research compared three different groups under three different conditions. Eight child-caregiver dyads were accompanied throughout Baseline 1 during three or four consultations, depending on specific hospitalization schedules of each child (totaling 31 consultations). Similarly, 20 child-caregiver dyads were accompanied during the Intervention phase over the course of one to four consultations, depending on specific hospitalization schedules (totaling 56 consultations). In Baseline 2, four child-caregiver dyads were accompanied for four consultations each (totaling 16 consultations), in all totaling 103 consultations.

The same child-caregiver dyads were not the same throughout the three phases of the study due to the treatment protocol, which required consultations to be spaced apart (usually fortnightly, and rarely once a week). This situation, added to the unforeseen deterioration in the clinical status, unplanned hospitalization or clinical complications, usually lasting the first six months of cancer treatment, during which contact with treatment itself, the hospital environment, the health team, and other child-caregiver dyads undergoing advanced treatment, places a crucial interference in the participants' reinforcement history, as they receive a great amount of information outside the consultations, not provided by the physicians, only by being in contact with this new environment. Following-up the same child-caregiver dyads would, therefore, involve many intervenient variables from treatment itself, which could bias the observation of pre-consultation lists. In both Baselines, consultations were audio recorded, without any intervention, in order to evaluate the communicative behaviors of caregivers and children toward the pediatricians during the medical visits.

In the Intervention phase, caregivers and children were individually interviewed by a researcher while waiting for ambulatory consultations in the waiting room. During the interview, the researcher would ask the caregivers and children about the diagnosis, expectancies and treatment planning, adherence to medication and self care, doubts and difficulties related to treatment, and other issues that they would like to discuss with the physician during the consultation. These issues were written down by the researcher on the pre-consultation list, an instrument that would be immediately attached to the cover of the child's medical record. The list would then be available during the consultation, in order for the physicians, children and caregivers to use it as a memo during the medical visit and discuss the issues raised.

The data analysis was carried out as follows. The communicative behavior corresponded to any kind of verbal interaction from children or caregivers directed specifically to the physician during the consultation. Throughout the three phases of the research (Baseline 1, Intervention and Baseline 2), data were analyzed according to the communicative categories created exclusively for this study after listening to half of the data recorded, which was randomly chosen from each situation. With regard to the communicative behaviors from children, there were four categories: 1) protesting (child cries, yells and/or complains about the treatment); 2) doubts (child asks about treatment related issues); 3) socialization (child talks to the physician about issues not related to the treatment); and 4) information giving (child gives information about well being, symptoms or exams). As for the communicative behaviors from caregivers, there were five categories: 1) doubts (caregiver asks about 
treatment related issues); 2) difficulties (caregiver tells physician about personal difficulties); 3) information giving (caregiver gives information about child's treatment); 4) including child (caregiver asks and/or explains something to the child); and 5) socializations (caregiver talks to the physician about issues not related to the treatment).

Particularly in Intervention, data analysis was also organized according to three main antecedent events that may have promoted the communicative behaviors of caregivers and children: (a) direct verbal request and/or questions from physician; (b) use of pre-consultation list; or (c) other events. Direct verbal request from the physician corresponded to questions or direct demands for information, for example when the pediatrician asked "How is he doing? Any fever?" or "Do you have any questions?" and then the caregivers or children would answer and provide information to physicians. The use of pre-consultation lists corresponded to the moment when the physician, caregivers and/or children would refer to the pre-consultation lists, for example by reading the demands and doubts, and therefore the caregivers and children could talk about issues listed on the protocol.

Other events, which could not be directly observed in audio recordings, might have been related to verbal behaviors from caregivers and children. They would include, for example, spontaneous provision of information, asking questions and/or telling the physician about difficulties, without the pediatrician needing to ask directly or use of pre-consultation lists. Due to the number of consultations recorded, the KruskalWallis non-parametric tests were used in the statistical analysis ( $p$ values in Shapiro-Wilk's normality test were lower than 0.05 ). The inter-rater reliability of behavioral observations reached values between 75.7 and $95.0 \%$, with a mean of $84.55 \%$ (Standard Deviation - SD $=4.13$ ).

\section{Results}

This section presents the communicative behaviors of the children and caregivers, comparing the three study phases, in addition to the effects of pre-consultation lists on these interactions.

\section{Children's communicative behaviors}

Figure 1 shows the average of the children's communicative behaviors in the three study stages. It should be highlighted that throughout the three study phases, the children contributed mainly to socialization and provision of information. Protesting and doubts were 34 and 55\%, respectively, lower in Intervention when compared with Baseline 1. Socialization was 49\% lower between Baseline 1 and Intervention, but it was 53\% higher in Baseline 2 when compared with Intervention. Finally, provision of information was $287 \%$ higher in Intervention when compared with Baseline 1 and $48 \%$ higher between Intervention and Baseline 2. Doubts addressed by the children in all phases were related to hospital admission and treatment planning or self care, hygiene, leisure and food recommendations. Provision of information was mainly associated with well being, symptoms, exams and clinical condition.

A functional analysis concerning the effects of pre-consultation lists on the children's communicative behaviors showed that protesting, socialization and provision of information were not related to the procedure. However, during Intervention the preconsultation lists raised $17 \%$ of doubts related to hospital admission and treatment planning, and only $4 \%$ of these doubts were addressed after the

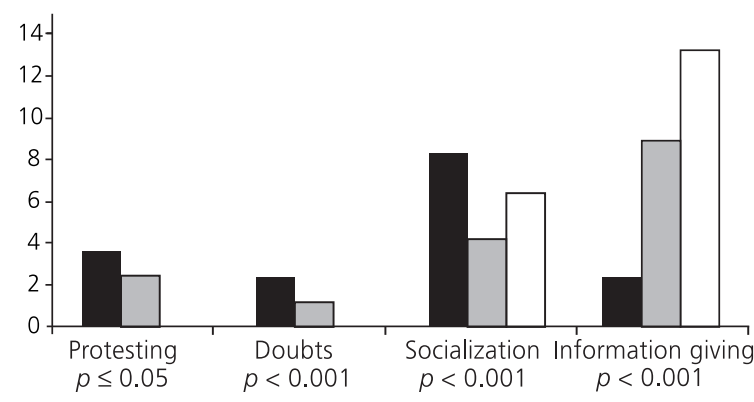

Baseline $1 \quad \square$ Intervention $\square$ Baseline 2

Figure 1. Average of children's communicative behaviors in the study phases. 
physician's questions $\left(\chi^{2}[2]=15.1 ; p=0.001\right)$. Besides that, pre-consultation lists during the Intervention phase raised $83 \%$ of doubts regarding self care, hygiene, leisure and feeding, while none of the pediatricians' questions were related to these kind of doubts $\left(\chi^{2}[2]=7.41 ; p=0.025\right)$. Overall, pre-consultation lists raised $30 \%$ of all doubts during Intervention, and only $3 \%$ of this behavior occurred at the pediatricians' request $\left(\chi^{2}\right.$ $[2]=13.21 ; p=0.001)$.

\section{Caregiver's communicative behavior}

Figure 2 shows the average of communicative behaviors from caregivers in the study stages. It is noteworthy that doubts were $99 \%$ higher during Intervention when compared with Baseline 1 and $25 \%$ lower in Baseline 2 when compared with Intervention. There were no relevant differences regarding the inclusion of the child in the communication between the groups in each phase. Provision of information was 29\% higher in Intervention when compared with Baseline 1, and $39 \%$ lower in Baseline 2 when compared with Intervention. Finally, socialization behaviors were $34 \%$ lower in Intervention when compared with Baseline 1 and 35\% lower in Baseline 2 when compared with Intervention.

Behaviors concerning the inclusion of the child in interaction and socialization were not

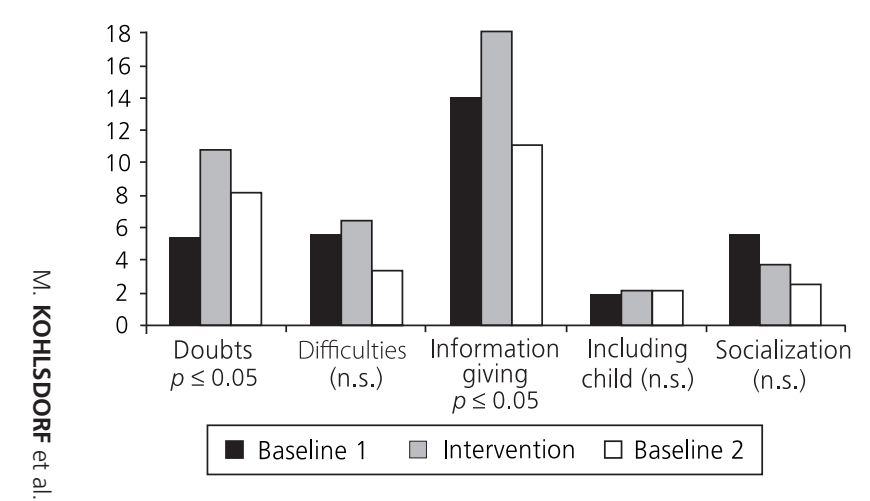

Figure 2. Average of caregivers' communicative behaviors in the study phases.

628

Note: n.s.: not significant. related to pre-consultation lists. In general, $70 \%$ of caregiver's doubts were related to other events, $27 \%$ were raised by pre-consultation lists and only $3 \%$ occurred due to the pediatrician's questions $\left(\chi^{2}[2]=82.52 ; p<0.001\right)$. Difficulties were mainly related to other events (42\%) or the physician's questions (28\%), but were also raised by preconsultation lists (30\%), although without any statistical significance $\left(\chi^{2}[2]=3.48 ; p=0.147\right)$. Finally, the provision of information was related to pediatrician's questions in $61 \%$ of the occurrences, $30 \%$ were due to other events and pre-consultation lists raised only $9 \%$ of this behavior $\left(\chi^{2}[2]=97.89\right.$; $p<0.001)$. However, in some specific visits, the pre-consultation lists seemed to have a greater influence on the caregiver's behavior. In eight consultations, the protocol was responsible for $45 \%$ to $100 \%$ of the caregivers' doubts, and, in nine visits, pre-consultation lists raised $60 \%$ to $100 \%$ of the difficulties addressed by the caregivers.

Table 2 shows the list of doubts, difficulties addressed and information provided by the caregivers throughout the three study phases. It is noteworthy that doubts about school activities and sequelae/relapse were only addressed by the group in Intervention condition. Besides that, during Intervention, doubts remained focused on feeding and food, self care, hygiene and leisure activities, coping with side effects, biological features of cancer and medication. Difficulties related to educational practices were also higher for the groups in the Intervention group, although without any statistical significance. Provision of information related to symptoms, clinical condition, food recommendations, exams, medication, admission, school and leisure activities were higher for those in the Intervention group.

Table 3 shows the functional analysis of specific issues related to doubts, difficulties and provision of information provided by the caregivers. It should be noted that the pre-consultation lists addressed a significant amount of doubts regarding feeding/food, coping with side effects, child development, biological features of cancer, sequelae or relapses, and school activities. However, the protocol was not directly related to difficulties 
Table 2

Doubts, difficulties addressed, and information provided by caregivers

\begin{tabular}{|c|c|c|c|}
\hline Doubts, difficulties addressed and information provided & Baseline 1 & Intervention & Baseline 2 \\
\hline Doubts: exams and procedures* & 1.03 & 2.29 & 3.3 \\
\hline Doubts: hospital admission, treatment planning & 1.97 & 2.45 & 1.8 \\
\hline Doubts: feeding and food ${ }^{*}$ & 0.26 & 0.95 & 0.3 \\
\hline Doubts: self care, hygiene and leisure activities & 0.13 & 0.59 & 0.4 \\
\hline Doubts: school activities ${ }^{* *}$ & - & 0.36 & - \\
\hline Doubts: coping with side effects* & 0.29 & 1.05 & 0.3 \\
\hline Doubts: child development & 0.10 & 0.09 & 0.1 \\
\hline Doubts: biological features of cancer & 0.32 & 0.59 & 0.3 \\
\hline Doubts: medication* & 0.52 & 1.13 & 0.4 \\
\hline Doubts: health service & 0.81 & 1.02 & 1.3 \\
\hline Doubts: sequelae and relapse* & - & 0.29 & - \\
\hline Difficulties: professional, financial, health-related service & 1.61 & 1.57 & 1.9 \\
\hline Difficulties: lack of social support & 0.55 & 0.75 & 0.3 \\
\hline Difficulties: treatment adherence & 0.39 & 0.46 & - \\
\hline Difficulties: educational practices & 1.52 & 2.48 & 1.1 \\
\hline Difficulties: emotional issues* ${ }^{*}$ & 1.55 & 1.09 & 0.1 \\
\hline Information on symptoms, clinical condition, feeding* & 6.97 & 8.64 & 5.4 \\
\hline Information on exams, medication and admission* & 6.68 & 7.71 & 5.1 \\
\hline Information on school and leisure activities* & 0.61 & 1.84 & 0.9 \\
\hline
\end{tabular}

Note: ${ }^{*} p \leq 0.05 ;{ }^{* *} p \leq 0.01$

Table 3

Effects of the pre-consultation lists on doubts, difficulties addressed, and information provided by caregivers

\begin{tabular}{|c|c|c|c|}
\hline Doubts, difficulties addressed and information provided & Other events & Pre-consultation lists & Physician's request \\
\hline Doubts: exams and procedures** & 84 & 14 & 2 \\
\hline Doubts: hospital admission, treatment planning ${ }^{* *}$ & 79 & 20 & 1 \\
\hline Doubts: feeding and food ${ }^{* *}$ & 23 & 70 & 7 \\
\hline Doubts: self care, hygiene and leisure* & 64 & 30 & 6 \\
\hline Doubts: school activities* & 60 & 40 & - \\
\hline Doubts: coping with side effects** & 48 & 52 & - \\
\hline Doubts: child development & 40 & 40 & 20 \\
\hline Doubts: biological features of cancer* & 61 & 27 & 12 \\
\hline Doubts: medication** & 86 & 14 & - \\
\hline Doubts: health service ${ }^{* *}$ & 93 & 5 & 2 \\
\hline Doubts: sequelae and relapse & 38 & 50 & 12 \\
\hline Difficulties: professional, financial, health service related & 41 & 30 & 29 \\
\hline Difficulties: lack of social support & 26 & 16 & 58 \\
\hline Difficulties: treatment adherence ${ }^{*}$ & 73 & 4 & 23 \\
\hline Difficulties: educational practices & 34 & 38 & 28 \\
\hline Difficulties: emotional issues ${ }^{* *}$ & 61 & 31 & 8 \\
\hline Information on symptoms, clinical condition, feeding ${ }^{* *}$ & 36 & 8 & 56 \\
\hline Information on exams, medication and admission ${ }^{* *}$ & 18 & 7 & 75 \\
\hline Information on school and leisure activities* & 51 & 21 & 28 \\
\hline
\end{tabular}

Note: ${ }^{*} p \leq 0.05 ;{ }^{* *} p \leq 0.01$.

addressed or provision of information. It is also possible that the pre-consultation lists may have changed the triadic dynamics in other non- observable ways that could have raised more doubts in caregivers, even without the direct influence of the procedure on communicative behavior. 


\section{Discussion}

Results show that some specific doubts from children were raised when the protocol was used, such as hospital admission or treatment planning and self care, hygiene, leisure and food recommendations; when considering the caregiver's demands, particular doubts were also focused on food recommendations, coping with side effects, child development, biological features of cancer, sequelae or relapses, and school activities.

Although there were no remarkable associations between pre-consultation lists and other communicative behaviors, such as provision of information and reported difficulties, the protocol might have helped focusing on individual demands of children and caregivers that usually are not included in common consultations, since it is a low-cost procedure that promotes the discussion of individual demands, as previously highlighted in the studies of Felt and O'Connor (2003) and Hayutin et al. (2009). Therefore, it may be a relevant tailored intervention to help improve communication during medical visits, according to specific demands of parents and children, which is in agreement with literature (Aburn \& Gott, 2011; Coyne \& Gallagher, 2011; Coyne \& Harder, 2011; Howells \& Lopez, 2008; Lambert et al., 2011; Ringnér et al., 2010; Zwaanswijk et al., 2011).

Results have shown that, throughout the three phases of the study, children mainly contributed to socialization and provision of information, as described in other studies (Tates \& Meeuwesen, 2000, 2001; Wissow \& Kimel, 2002). It is important to point out that pre-consultation lists may help address the children's demands during consultations, perhaps indirectly when focusing on provision of information, as found by the differences between the three phases of the study. The literature highlights the need for including children as active participants in the health-related processes and the importance of the psychosocial interventions for increasing patient's participation (Coyne, 2008; Hallström, 2004; Nobile \& Drotar, 2003; Nova et al., 2005; Rotenberg et al., 2008; Tates \& Meeuwesen, 630 2000, 2001; Tates et al., 2002a, 2002b; Van Dulmen,
2004; Van Dulmen \& Holl, 2000; Vaknin \& ZiskRony, 2010; Wissow \& Kimel, 2002).

Further studies should investigate the role of these interventions using pre-consultation lists on future health-related behaviors, as the literature points out the relevant association among good communication and self-care behavior, quality of life, adaptation to treatment, satisfaction with health services, remembering instructions, improving symptoms and physiological responses, management of stressors concerning health processes, better social support, adherence to treatment and fewer ambulatory returns (Ammentorp et al., 2011; Clark et al., 2000; Cohen \& Wambolt, 2000; Coyne \& Gallagher, 2011; Croom et al., 2011; Crossley \& Davies, 2005; DiMatteo, 2004; Drotar, 2009; Howells \& Lopez, 2008; Nobile \& Drotar, 2003; Sleath et al., 2012). For example, further studies could investigate the children's and caregivers' satisfaction with the pre-consultation procedures, compare pediatric groups that used pre-consultation lists concerning their adherence to the treatment of chronic conditions such as asthma or diabetes, analyze emergency returns related to health complications in groups undergoing cancer treatment that were exposed to the procedure, or to understand the point of view of the pediatricians regarding these lists.

\section{Final Considerations}

This research has some limitations that must be discussed. First, the direct observation of medical visits is relevant to investigate how the triadic interaction actually occurs, however it is possible that the pre-consultation lists had effects which could not be measured since they were related to non- observable private behaviors. For instance, it is possible that the increase of doubts of the caregivers during Intervention could have been related to pre-consultation interviews, which would suggest new topics and treatment issues for discussion, or even change the communication dynamics in order to promote more verbalization from caregivers. However, this could not be directly addressed. 
Second, more participants would be required to endorse the data obtained as only 32 childcaregiver dyads participated in the research, thus the results must be interpreted carefully. However, all eligible child-caregiver dyads were invited to participate in the study and it was not possible to include more participants due to inclusion criteria. Furthermore, the characteristics of the childcaregiver dyads regarding age, schooling, and socioeconomical background were not controlled and some differences in these characteristics were statistically significant, which could have influenced the communicative patterns. Third, more consultations would have been interesting. Nevertheless, due to cancer treatment planning, it was not possible to record a larger number of medical visits. Even within these limitations, the study contributes to pediatric psychology by proposing a low-cost procedure that may improve pediatric communication during medical visits by tailoring the interaction to individual needs and including children in the communicative process. Further studies are needed to deepen our understanding of the effects of preconsultation lists and other psychosocial interventions on pediatric communication.

\section{Contributors}

M. KOHLSDORF and A.L. COSTA JUNIOR developed the experimental design, carried out data analysis and drafted the manuscript. M. KOHLSDORF and F.D. MARQUES also carried out the data collection. All authors read and approved the final manuscript.

\section{References}

Aburn, G., \& Gott, M. (2011). Education given to parents of children newly diagnosed with acute lymphoblastic leukemia: A narrative review. Journal of Pediatric Oncology Nursing, 28(5), 300-305.

Ammentorp, J., Kofoed, P. E., \& Laulund, L. W. (2011). Impact of communication skills training on parents perceptions of care: Intervention study. Journal of Advanced Nursing, 67(2), 394-400.

Araújo, I. S., \& Cardoso, J. M. (2007). Comunicação e saúde. Rio de Janeiro: Fiocruz .

Arora, N. K. (2003). Interacting with cancer patients: The significance of physician's communication behavior. Social Science \& Medicine, 57(5), 791-806.
Buckley, A., \& Savage, E. (2010). Preoperative information needs of children undergoing tonsillectomy. Journal of Clinical Nursing, 19(19-20), 2879-2887.

Clark, N. M., Gong, M., Schork, M. A., Kaciroti, N., Evans, D., Roloff, D., ... Mellins, R. B. (2000). Long-term effects of asthma education for physicians on patient satisfaction and use of health services. European Respiratory Journal, 16(1), 15-21.

Cohen, S. Y., \& Wamboldt, F. S. (2000). The parentphysician relationship in pediatric asthma care. Journal of Pediatric Psychology, 25(2), 69-77.

Coyne, I. (2008). Children's participation in consultations and decision-making at health service level: A review of the literature. International Journal of Nursing Studies, 45(11), 1682-1689.

Coyne, I., \& Gallagher, P. (2011). Participation in communication and decision-making: Children and young people's experiences in a hospital setting. Journal of Clinical Nursing, 20(15-16), 2334-2343.

Coyne, I., \& Harder, M. (2011). Children's participation in decision-making: Balancing protection with shared decision-making using a situational perspective. Journal of Child Health Care, 15(4), 312-319.

Croom, A., Wiebe, D. J., Berg, C. A., Lindsay, R., Donaldson, D., Foster, C., ... Swinyard, M. T. (2011). Adolescent and parent perceptions of patient-centered communication while managing type 1 diabetes. Journal of Pediatric Psychology, 36(2), 206-215.

Crossley, J., \& Davies, H. (2005). Doctor's consultation with children and their parents: A model of competencies, outcomes and confounding influences. Medical Education, 39(8), 807-819.

DiMatteo, M. R. (2004). The role of effective communication with children and their families in fostering adherence to pediatric regimens. Patient Education and Counseling, 55(3), 339-344.

Drotar, D. (2009). Physician behavior in the care of pediatric chronic illness: Association with health outcomes and treatment adherence. Journal of Developmental \& Behavioral Pediatrics, 30(3), 246-254.

Felt, B. T., \& O'Connor, M. E. (2003). Use of the child development review increases residents' discussion of behavioral problems. Ambulatory Pediatrics, 3(1), 2-8.

Fisher, M. J., \& Broome, M. E. (2011). Parent-provider communication during hospitalization. Journal of Pediatric Nursing, 26(1), 58-69.

Gabe, J., Olumide, G., \& Bury, M. (2004). 'It takes three to tango': A framework for understanding patient partnership in paediatric clinics. Social Science and Medicine, 59(5), 1071-1079.

Gordon, B. K., Jaaniste, T., Bartlett, K., Perrin, M., Jackson, A., Sandstrom, A., ... Seehan, S. (2010). Child and parental surveys about pre-hospitalization information provision. Child: Care, Health and Development, 37(5), 727-733. 
Hallström, I. (2004). Parents' and children's involvement in decision-making during hospitalization. Nursing Times Research, 9(4), 263-269.

Hayutin, L. G., Reed-Knight, B., Blount, R. L., Lewis, J., \& McCormick, M. L. (2009). Increasing parent-pediatrician communication about children's psychosocial problems. Journal of Pediatric Psychology, 34(10), 1155-1164.

Howells, R., \& Lopez, T. (2008). Better communication with children and parents. Paediatrics and Child Health, 18(8), 381-385.

Howells, R. J., Davies, H. A., \& Silverman, J. D. (2006). Teaching and learning consultation skills for paediatric practice. Archives of Disease in Childhood, 91(4), 367-370.

Knighting, K., Rowa-Dewar, N., Malcolm, C., Kearney, N., \& Gibson, F. (2010). Children's understanding of cancer and health behaviors. Child: Care, Health and Development, 37(2), 289-299.

Lambert, V., Glacken, M., \& McCarron, M. (2011). Communication between children and health professionals in a child hospital setting: A child transitional communication model. Journal of Advanced Nursing, 67(3), 569-582.

Märtenson, E. K., \& Fägerskiöld, A. M. (2007). A review of children's decision-making competence in health care. Journal of Clinical Nursing, 17(23), 3131-3141.

Märtenson, E. K., Fägerskiöld, A. M., \& Berteró, C. M. (2007). Information exchange in paediatric settings: An observational study. Paediatric Nursing, 19(7), 40-43.

McGraw, M., Fellows, S., Long, A., Millar, H., Muir, G., Thomson, A., ... Williams, S. (2012). Feedback on doctors' performance from parents and carers of children: A national pilot study. Archives of Disease in Childhood, 97(3), 206-210.

Nobile, C., \& Drotar, D. (2003). Research on the quality of parent-provider communication in pediatric care: Implications and recommendations. Developmental and Behavioral Pediatrics, 24(4), 279-290.

Nova, C., Vegni, E., \& Moja, E. A. (2005). The physicianpatient-parent communication: A qualitative perspective on the child's contribution. Patient Education and Counseling, 58(3), 327-333.

Ringnér, A., Jansson, L., \& Graneheim, U. H. (2010). Professional caregivers' perceptions of providing information to parents of children with cancer. Journal of Pediatric Oncology Nursing, 20(10), 1-9.

Rotenberg, K. J., Cunningham, J., Hayton, N., Hutson, L., Jones, L., Marks, C., ... Betts, L. R. (2008). Development of a children's trust in general physicians scale. Child: Care, Health and Development, 34(6), 748-756.

Sleath, B., Carpenter, D. M., Slota, C., Williams, D., Tudor, 632 G., Yeatts, K., ... Ayala, G. X. (2012). Communication during pediatric asthma visits and self-reported asthma medication adherence. Pediatrics, 130(4), 1-7.

Tates, K., \& Meeuwesen, L. (2000). "Let mum have her say": Turntaking in doctor-parent-child communication. Patient Education and Counseling, 40(2), 151-162.

Tates, K., \& Meeuwesen, L. (2001). Doctor-parent-child communication: A (re)view of the literature. Social Science and Medicine, 52(6), 839-851.

Tates, K., Elbers, E., Meeuwesen, L., \& Bensing, J. (2002a). Doctor-parent-child relationships: A 'pas de trois'. Patient Education and Counseling, 48(1), 5-14.

Tates, K., Meeuwesen, L., Elbers, E., \& Bensing, J. (2002b). "I've come for his throat": Roles and identities in doctor-parent-child communication. Child: Care, Health, \& Development, 28(1), 109-116.

Vaknin, O., \& Zisk-Rony, R. Y. (2010). Including children in medical decisions and treatments: Perceptions and practices of healthcare providers. Child: Care, Health and Development, 37(4), 533-539.

Van Dulmen, S. (2004). Pediatrician-parent-child communication: Problem-related or not? Patient Education and Counseling, 52(1), 61-68.

Van Dulmen, S., \& Holl, R. A. (2000). Effects of continuing paediatric education in interpersonal communication skills. European Journal of Pediatrics, 159(7), 489-495.

Vatne, T. M., Slaughter, L., \& Ruland, C. M. (2010). How children with cancer communicate and think about symptoms. Journal of Pediatric Oncology Nursing, 27(1), 24-32.

Wassmer, E., Minnaar, G., Aal, N. A., Atkinson, M., Gupta, E., Yuen, S., \& Rylance, G. (2004). How do paediatricians communicate with children and parents? Acta Paediatrica, 93(11), 1501-1506.

Wissow, L. S., \& Kimel, M. B. (2002). Assessing providerpatient-parent communication in the pediatric emergency department. Ambulatory Pediatrics, 2(4), 323-329.

Zannon, C. M. L. C., Pereira, R. M. G., Arruda, P. M., Kohlsdorf, M., \& Rocha L. E. P. (2002). Pré-consulta comportamental: identificando problemas e promovendo aliança para o tratamento. Revista Brasileira de Reumatologia, 14(19), 79.

Zwaanswijk, M., Tates, K., van Dulmen, S., Hoogerbrugge, P. M., Kamps, W. A., Beishuizen, A., \& Bensing, J. M. (2011). Communicating with child patients in pediatric oncology consultations: A vignette study on child patients', parents', and survivors' communication preferences. Psycho-Oncology, 20(3), 269-277.

Received: March 3, 2014

Final version: August 8, 2014 Approved: October 30, 2014 